\title{
Humoral and cellular immune responses in dogs with inapparent natural Leishmania infantum infection is
}

\author{
W. Coura-Vital ${ }^{\text {a,b }}$, M.J. Marques ${ }^{c}$, R.C. Giunchetti ${ }^{\text {a,d }}$, A. Teixeira-Carvalho ${ }^{\text {d }}$, N.D. Moreira ${ }^{\text {a }}$, \\ J. Vitoriano-Souza ${ }^{\text {a }}$, P.M. Vieira ${ }^{\text {a }}$, C.M. Carneiro ${ }^{\text {a }}$, R. Corrêa-Oliveira ${ }^{\mathrm{e}}$, O.A. Martins-Filho ${ }^{\mathrm{d}}$, \\ M. Carneiro ${ }^{\mathrm{b}}$, A.B. Reis ${ }^{\mathrm{a}, \mathrm{e}, *}$ \\ ${ }^{a}$ Laboratório de Imunopatologia, Núcleo de Pesquisas em Ciências Biológicas/NUPEB, Universidade Federal de Ouro Preto, Ouro Preto, Minas Gerais, Brazil

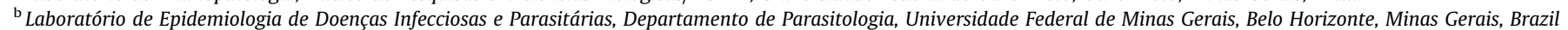

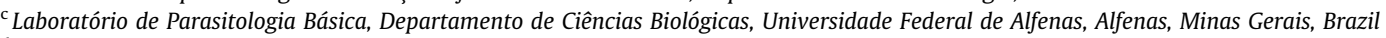 \\ ${ }^{\mathrm{d}}$ Laboratório de Biomarcadores de Diagnóstico e Monitoração, Centro de Pesquisas René Rachou, Fundação Oswaldo Cruz, Belo Horizonte, Minas Gerais, Brazil \\ e Laboratório de Imunologia Celular e Molecular, Centro de Pesquisas René Rachou, Fundação Oswaldo Cruz, Belo Horizonte, Minas Gerais, Brazil
}

\section{A R T I C L E I N F O}

\section{Article history:}

Accepted 7 April 2011

\section{Keywords:}

Canine visceral leishmaniasis

Asymptomatic dogs

Immune response

\begin{abstract}
A B S T R A C T
Molecular analysis, serology and immunophenotyping for T lymphocytes and their subsets, B lymphocytes and monocytes were performed on dogs naturally infected with Leishmania infantum. Animals were categorised as asymptomatic dogs I (AD-I), with negative serology and positive molecular results, and asymptomatic dogs II (AD-II), with positive serology and positive molecular results, and these were compared to symptomatic dogs (SD) and control dogs (CD).

AD-I exhibited immunophenotypic features similar to those of $C D$, including isotype profiles and concentrations of monocytes. Similar biomarkers were found in AD-II and SD, such as, higher levels of immunoglobulins IgG, IgG2, IgM and IgA and higher concentrations of eosinophils. High frequencies of T lymphocytes and $\mathrm{CD} 4^{+} \mathrm{T}$ cells were observed in both $\mathrm{AD}-\mathrm{I}$ and $\mathrm{AD}-\mathrm{II}$ compared to $\mathrm{SD}$, whereas $\mathrm{CD} 8^{+} \mathrm{T}$ cells were higher only in AD-II compared with SD. Analysis of B lymphocytes revealed an increased frequency of this cell type only in AD-II animals compared with SD. Asymptomatic dogs appear to have a dichotomous infection spectrum that can influence the humoral and cellular immunological status during canine visceral leishmaniasis.
\end{abstract}

(c) 2011 Elsevier Ltd. All rights reserved.

\section{Introduction}

Leishmaniasis is endemic in 88 countries in tropical and subtropical regions of the Old and New World, with more than 350 million people exposed to the infection (Desjeux, 2001). The estimated incidence is 2 million new cases per year, with 0.5 million being visceral leishmaniasis (VL) (Desjeux, 2004). Infected dogs have a high cutaneous parasite density and represent the main domestic reservoir of Leishmania infantum, contributing to the propagation of the disease (Deane and Deane, 1962). In Brazil over the past 10 years, more than 2 million dogs were screened and more than 160,000 seropositive dogs were eliminated; however, the incidence of human VL has not been reduced to an acceptable level (Lemos et al., 2008; Romero and Boelaert, 2010).

Canine visceral leishmaniasis (CVL) can be categorised into three distinct clinical forms on the basis of major features observed

\footnotetext{
This article is available only in the online version of this issue of The Veterinary Journal at http://www.sciencedirect.com/science/journal/10900233

* Corresponding author. Tel.: +55 213135591694 .

E-mail address: alexreis@nupeb.ufop.br (A.B. Reis).
}

in seropositive infected dogs, which can be classified as asymptomatic dogs (AD), oligosymptomatic dogs and symptomatic dogs (SD) dogs (Mancianti et al., 1988; Reis et al., 2009). L. infantum-infected dogs also include animals in which the presence of parasites is confirmed through direct methods (such as PCR) and which have lowtitre anti-Leishmania antibodies (Paltrinieri et al., 2010).

Oliva et al. (2006) identified subpatent infection with L. infantum in asymptomatic dogs that were intermittently positive by nested PCR but became negative for long periods of time. Seropositive asymptomatic dogs are important sources of amastigotes for infection of phlebotomines that contribute to the transmission of L. infantum (Marzochi et al., 1985; Molina et al., 1994; da CostaVal et al., 2007). In endemic areas, $10-62 \%$ of apparently healthy and/or seronegative dogs were positive for Leishmania by PCR (Martin-Sanchez et al., 2001; Solano-Gallego et al., 2001; Lachaud et al., 2002; Andrade et al., 2006). Thus, asymptomatic dogs may play a role in the transmission of Leishmania parasites but cannot be detected by conventional serological tests, such as the indirect fluorescent antibody test (IFAT) and enzyme-linked immunosorbent assay (ELISA). 
The aim of this study was to use the humoral and cellular immune response to explore the dichotomy in dogs with asymptomatic and symptomatic infection with $L$. infantum and to identify features that could be used to identify resistant and susceptible profiles.

\section{Materials and methods}

\section{Dogs and experimental design}

Forty-one mongrel dogs of either gender from the endemic area of Belo Horizonte, Minas Gerais, Brazil, were selected on the basis of serological tests (IFAT and ELISA, Biomanguinhos/Fiocruz) for Leishmania spp. Dogs with an IFAT titre $<1 / 40$ were considered to be seronegative and dogs with an IFAT titre $\geqslant 1 / 40$ were considered to be seropositive and infected with Leishmania spp. Positive infection was confirmed by ELISA and PCR in at least one skin sample (Degrave et al., 1994) and the species of Leishmania responsible was determined by restriction fragment length polymorphism-PCR (Volpini et al., 2004).

The study was conducted from June 2008 to August 2009 after approval by the ethical committees for the use of experimental animals of the Federal University of Ouro Preto (CETEA/UFOP 032/2007), Federal University of Minas Gerais (CETEA/ UFMG 020/2007) and the Municipal Health Secretariat of Belo Horizonte City Council, Minas Gerais State, Brazil (CEP-SMSA/PBH 001/2008).

\section{Selection and clinical classification of dogs}

Dogs were selected and clinically classified according to the presence/absence of clinical signs: (1) asymptomatic, with no signs suggestive of disease; (2) symptomatic, with characteristic clinical signs of visceral leishmaniasis, such as opaque bristles, severe loss of weight, onychogryphosis, cutaneous lesions, apathy and keratoconjunctivitis; and (3) control dogs, classified according to negative serological and molecular results and absence of clinical signs.

\section{Haematology}

Peripheral blood $(5 \mathrm{~mL})$ from the brachiocephalic vein was collected into tubes containing ethylene diamine tetraacetic acid (EDTA) at a final concentration of $1 \mathrm{mg} / \mathrm{mL}$. Erythrocytes and leucocytes were quantified using an automatic cell counter (Model 2800 Vet, Mindray). Differential leucocyte counts were performed by examination of at least 200 leucocytes in Giemsa-stained blood smears by light microscopy.

\section{ELISA for immunoglobulin isotype profile}

ELISAs were performed to determine the anti-Leishmania immunoglobulin pattern using soluble L. infantum (MHOM/BR/1972/BH46) promastigotes antigen (SLA) (Rosário et al., 2005; Reis et al., 2006c). The protein concentration was quantified by the Lowry method, adjusted to $1 \mathrm{mg} / \mathrm{mL}$ and samples were stored at $-70{ }^{\circ} \mathrm{C}$. Ninety-six well microplates (MaxiSorp, Nalge Nunc) were coated with SLA at a concentration of $2 \mu \mathrm{g} /$ well, left overnight at $4-8{ }^{\circ} \mathrm{C}$ and then washed. Serum samples were added to the wells at a dilution of $1: 80$, followed by washes after the addition of goat anti-dog IgG1 (anti-heavy chain specific) conjugated with peroxidase, IgM (anti- $\mu$ chain specific), IgA (anti- $\alpha$ chain specific) and IgE (anti- $\varepsilon$ chain specific) or sheep anti-dog IgG and IgG2 (both anti-heavy chain specific) (Bethyl Laboratories). Wells were washed and then the substrate and chromogen (O-phenylenediamine, Sigma-Aldrich) were added. The absorbance was read on an automatic ELISA microplate reader (EL $800 \mathrm{G}$ PC, Bio-Tek) at $492 \mathrm{~nm}$. The concentrations of conjugate were determined by a block titration method with positive and negative standard sera. The conjugates anti-IgG1, IgM, IgA, IgE were used at a dilution of 1:1,000, antiIgG was used at a dilution of 1:8,000 and anti-IgG2 was used at a dilution of $1: 16,000$.

\section{Immunophenotyping by flow cytometry}

Immunophenotyping of peripheral blood by flow cytometry was performed as described by Reis et al. (2005). After a pre-fixation step, erythrocytes were lysed in $1 \mathrm{~mL}$ EDTA-treated whole blood by the slow addition of $13 \mathrm{~mL}$ fluorescence-activated cell sorter (FACS) lysing solution (Becton Dickinson), followed by incubation for $10 \mathrm{~min}$ at room temperature (RT). After centrifugation ( $450 \mathrm{~g}$ for $10 \mathrm{~min}$ at RT), the pellet was resuspended in $500 \mathrm{~mL}$ phosphate-buffered saline (PBS) supplemented with $10 \%$ fetal bovine serum (PBS-10\% FBS). Using 96-well U-bottom plates (Limbro Biomedicals), $30 \mu \mathrm{L}$ prefixed leucocyte suspension were incubated at RT for $30 \mathrm{~min}$ in the dark with $30 \mu \mathrm{L}$ monoclonal antibodies (mAbs) diluted previously in PBS-10\% FBS.

The mAbs used in the study were diluted purified anti-canine CD5 (1:800, rat IgG2a, clone YKIX322.3), anti-canine CD4 (1:1,000, rat IgG2a, clone YKIX302.9) and anti-canine CD8 (1:800, rat IgG1, clone YCATE55.9) (Serotec). Undiluted fluorescein isothiocyanate (FITC)-labelled mouse anti-human CD21 $(5 \mu \mathrm{L}$, mouse
IgG1, clone IOBla; Immunotech) and diluted phycoerythrin (PE)/Cy-5-conjugated mouse anti-human CD14 (50 $\mu \mathrm{L}, 1: 200$, mouse IgG2a, clone TÜK4; Serotec) were also used in direct immunofluorescence procedures. Cells were also incubated in the same conditions in the presence of $60 \mu \mathrm{L}$ of diluted FITC-conjugated sheep anti-rat IgG polyclonal antibody (1:100 or 1:200; Serotec).

Before flow cytometric data collection and analysis were performed, labelled cells were fixed for 30 min with $200 \mu \mathrm{L}$ FACS fix solution $(10.0 \mathrm{~g} / \mathrm{L}$ paraformaldehyde, $10.2 \mathrm{~g} / \mathrm{L}$ sodium cacodylate, $6.6 \mathrm{~g} / \mathrm{L}$ sodium chloride; $\mathrm{pH}$ 7.2). Flow cytometric measurements were performed on a FACScan (Becton Dickinson) and analysed using CellQuest software (10,000 events acquired per sample). The results were expressed in absolute counts (cell number $/ \mathrm{mm}^{3}$ ) through the product of the percentage of positive cells $\left(\mathrm{CD}^{+}, \mathrm{CD} 4^{+}, \mathrm{CD}^{+}\right.$and $\left.\mathrm{CD} 21^{+}\right)$within gated lymphocytes by absolute lymphocyte counts. Absolute counts for $\mathrm{T}$ lymphocyte subsets were also calculated as the sum of absolute values of $\mathrm{CD}^{+}$plus $\mathrm{CD} 8^{+}$cells. The absolute counts for monocytes were the products of $\mathrm{CD} 14^{+}$cells within ungated leucocytes by the total leucocyte counts.

\section{Statistical analysis}

Statistical analysis was performed using GraphPad Prism 5.0. The normality of the data was assessed using the Kolmogorov-Smirnoff test. Considering the nonparametric nature of all data sets, Kruskal-Wallis tests were used to investigate differences between the four groups, followed by Dunn's test for pairwise comparisons. Differences were considered to be significant at $P<0.05$.

\section{Results}

\section{Reclassification according to serological, molecular and clinical features}

Dogs with no clinical signs and negative serological and molecular results were included in the control group $(C D, n=7)$. Seronegative dogs without clinical signs but positive molecular results for L. infantum were classified as asymptomatic dogs I (AD-I, $n=8$ ). Dogs with positive serology and molecular results for $L$. infantum but no clinical signs were classified as asymptomatic dogs II (ADII, $n=10)$. Dogs with clinical signs and positive serological and molecular results were classified as symptomatic dogs (SD) $(n=16)$ (Table 1$)$.

\section{Haematology}

There were lower concentrations of eosinophils in AD-II and SD compared with AD-I and CD and lower concentrations of lymphocytes in SD compared with AD-II (Table 2). Symptomatic L. infantum infection was associated with decreased erythrocyte counts and haematocrits in AD-II compared with AD-I and CD $(P<0.05)$ and decreased haemoglobin concentrations in SD compared to other groups $(P<0.05)$.

\section{Anti-Leishmania immunoglobulin isotypes}

Concentrations of IgG, IgG1, IgG2, IgM, IgA and IgE in AD-I were similar to $C D$ (Fig. 1). There were increased IgG, IgG2, IgM and IgA concentrations in $\mathrm{AD}-\mathrm{II}$ and $\mathrm{SD}$ compared with $\mathrm{AD}-\mathrm{I}$ and $\mathrm{CD}$

Table 1

Serological and molecular status of dogs categorised according to clinical status with and without natural infection by Leishmania infantum.

\begin{tabular}{lcccccc}
\hline \multirow{2}{*}{ Groups } & \multicolumn{2}{l}{$\begin{array}{l}\text { Serodiagnosis } \\
\text { IFAT and ELISA }\end{array}$} & & \multicolumn{2}{l}{$\begin{array}{l}\text { Molecular diagnosis } \\
\text { PCR-RFLP }\end{array}$} & Total \\
\cline { 2 - 3 } \cline { 5 - 6 } & Positive & Negative & & Positive & Negative & \\
\hline Control (CD) & 0 & 7 & & 0 & 7 & 7 \\
Asymptomatic I (AD-I) & 0 & 8 & & 8 & 0 & 8 \\
Asymptomatic II (AD-II) & 10 & 0 & & 10 & 0 & 10 \\
Symptomatic (SD) & 16 & 0 & & 16 & 0 & 16 \\
Total & 26 & 15 & & 34 & 7 & 41 \\
\hline
\end{tabular}

IFAT, Indirect fluorescent antibody test; ELISA, Enzyme-linked immunosorbent assay; PCR-RFLP, Polymerase chain reaction-restriction fragment length polymorphism. 
Table 2

Haematological parameters of naturally infected with Leishmania infantum and uninfected dogs.

\begin{tabular}{|c|c|c|c|c|}
\hline \multirow[t]{2}{*}{ Haematological parameter } & \multicolumn{4}{|c|}{ Clinical groups } \\
\hline & $\mathrm{CD}$ & AD-I & AD-II & SD \\
\hline Erythrocytes (million $/ \mathrm{mm}^{3}$ ) & $6.8 \pm 0.4$ & $6.7 \pm 1.0$ & $5.8 \pm 0.7$ & $4.2 \pm 1.3^{\mathrm{a}, \mathrm{b}}$ \\
\hline Haemoglobin $(\mathrm{g} \%)$ & $17.0 \pm 0.6$ & $14.8 \pm 2.3$ & $14.1 \pm 2.3$ & $9.8 \pm 2.9^{\mathrm{a}, \mathrm{b}, \mathrm{c}}$ \\
\hline Haematocrit (\%) & $49.1 \pm 2.0$ & $44.0 \pm 6.7$ & $40.7 \pm 7.1$ & $28.6 \pm 8.2^{\mathrm{a}, \mathrm{b}}$ \\
\hline Leucocytes $\left(\times 10^{3} / \mathrm{mm}^{3}\right)$ & $13.4 \pm 3.5$ & $12.0 \pm 2.6$ & $13.0 \pm 4.5$ & $10.2 \pm 3.9$ \\
\hline Granulocytes & $10.1 \pm 3.6$ & $8.2 \pm 3.3$ & $7.4 \pm 3.1$ & $8.0 \pm 3.2$ \\
\hline Neutrophils & $7.9 \pm 3.8$ & $6.6 \pm 3.1$ & $6.5 \pm 2.7$ & $7.2 \pm 3.0$ \\
\hline Eosinophils & $2.1 \pm 0.8$ & $1.7 \pm 0.6$ & $0.5 \pm 0.3^{\mathrm{a}, \mathrm{b}}$ & $0.5 \pm 0.4^{\mathrm{a}, \mathrm{b}}$ \\
\hline Lymphocytes & $2.1 \pm 0.8$ & $3.0 \pm 1.9$ & $4.5 \pm 3.3$ & $1.7 \pm 1.0^{\mathrm{c}}$ \\
\hline Monocytes & $1.1 \pm 0.6$ & $1.0 \pm 0.5$ & $1.0 \pm 0.6$ & $0.5 \pm 0.4^{\mathrm{a}, \mathrm{b}}$ \\
\hline
\end{tabular}

Results are shown as the average values \pm standard deviation.

CD, Control dogs; AD-I, Asymptomatic dogs I; AD-II, Asymptomatic dogs II; SD, Symptomatic dogs.

a Statistically significant differences compared with $\mathrm{CD}$.

b Statistically significant differences compared with AD-I.

c Statistically significant differences compared with AD-II.

$(P<0.05)$. Concentrations of IgE were lower in AD-I compared with SD $(P<0.05)$. There were no significant differences in concentrations of IgG1 between groups.

\section{Immunophenotyping of circulating I lymphocytes and their subsets}

AD-I and AD-II had increased concentrations of T lymphocytes compared with SD $(P<0.05)$. AD-I and AD-II had higher counts of $\mathrm{CD}^{+} \mathrm{T}$ lymphocytes than SD, whereas AD-II, but not AD-I, had higher counts of $\mathrm{CD}^{+} \mathrm{T}$ lymphocytes than $\mathrm{CD}$ and $\mathrm{SD}$. There was a decreased ratio of $\mathrm{CD}^{+}: \mathrm{CD}^{+}$in $\mathrm{AD}-\mathrm{II}$ and $\mathrm{SD}$ as compared with CD (Fig. 2).

Immunophenotyping of circulating B lymphocytes and monocytes

AD-II and CD had higher concentrations of $\mathrm{CD} 21^{+} \mathrm{B}$ lymphocytes than SD (Fig. 3). AD-II and SD had lower concentrations of monocytes than AD-I and CD.

\section{Discussion}

In the current study, we identified lower concentrations of eosinophils in AD-II and SD compared with AD-I and CD. Decreased concentrations of eosinophils have been identified previously in Leishmania-infected dogs with clinical signs (Reis et al., 2006a), as well as in dogs with medium and high parasite densities in spleen and skin (Guerra et al., 2009).

AD-II and SD had higher levels of IgG, IgG2, IgM and IgA than AD-I or CD, whereas AD-I and CD had similar immunoglobulin isotype profiles. These findings suggest that AD-I are in the initial phase of CVL, whereas AD-II are unable to control the parasitism, as indicated by intense polyclonal activation (Reis et al., 2006c; Teixeira Neto et al., 2010). None of these isotypes can confer protective immunity against $L$. infantum.

High concentrations of IgG2 are associated with clinical signs in dogs with leishmaniasis (Bourdoiseau et al., 1997; Cavaliero et al., 1999; Boceta et al., 2000; Solano-Gallego et al., 2000; Cardoso et al., 2007), whereas high concentrations of IgG1 are present in asymptomatic carriers (Reis et al., 2006c). Although IgM usually is considered to be an immunological marker for the acute phase of parasitic disease, it has been demonstrated that this immunoglobulin also can be detected during the chronic phases of CVL (Genaro et al., 1992; Reis et al., 2006c; Rodriguez-Cortes et al., 2007). Reis et al. (2006c) suggest that IgA reactivity is a marker for tissue parasite density in CVL. SD had higher concentrations of IgE than AD-I. Increased levels of IgE have been associated with severe forms of the disease and elevated tissue parasite densities,

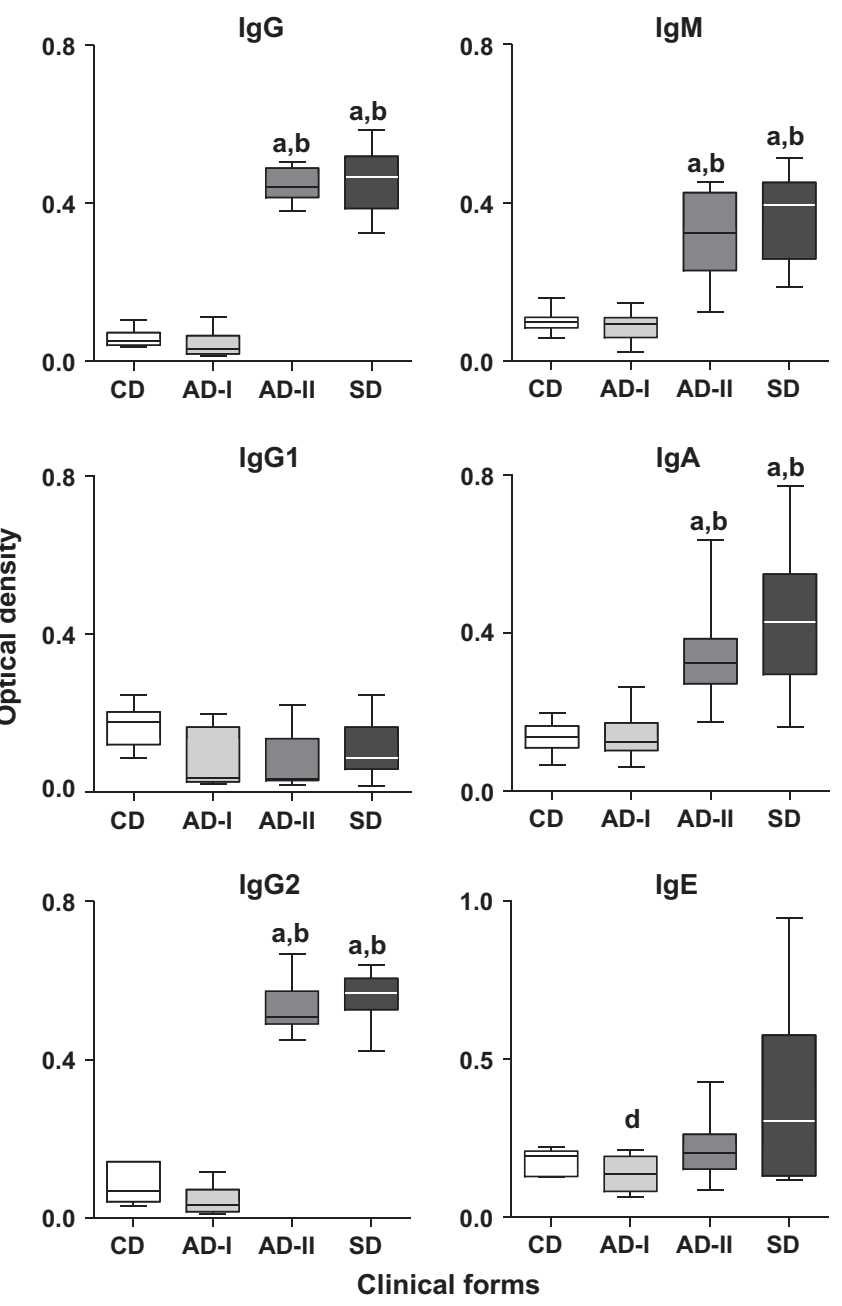

Fig. 1. Anti-Leishmania immunoglobulin isotype reactivities in dogs naturally infected with Leishmania infantum, categorised according to their clinical status as asymptomatic dogs I (AD-I), asymptomatic dogs II (AD-II) and symptomatic dogs (SD). Uninfected dogs were used as a control group (CD). The results are expressed as optical density values for each immunoglobulin isotype in box plot format highlighting the gap of $50 \%$ of data set measurement and the median and maximum and minimum values. ${ }^{\mathrm{a}, \mathrm{b}, \mathrm{d}}$ Significant differences at $P<0.05$ compared with $\mathrm{CD}, \mathrm{AD}-\mathrm{I}$ and $\mathrm{SD}$, respectively.

as well as with the T helper type 2 immune response profile (Reis et al., 2006c; Guerra et al., 2009). 

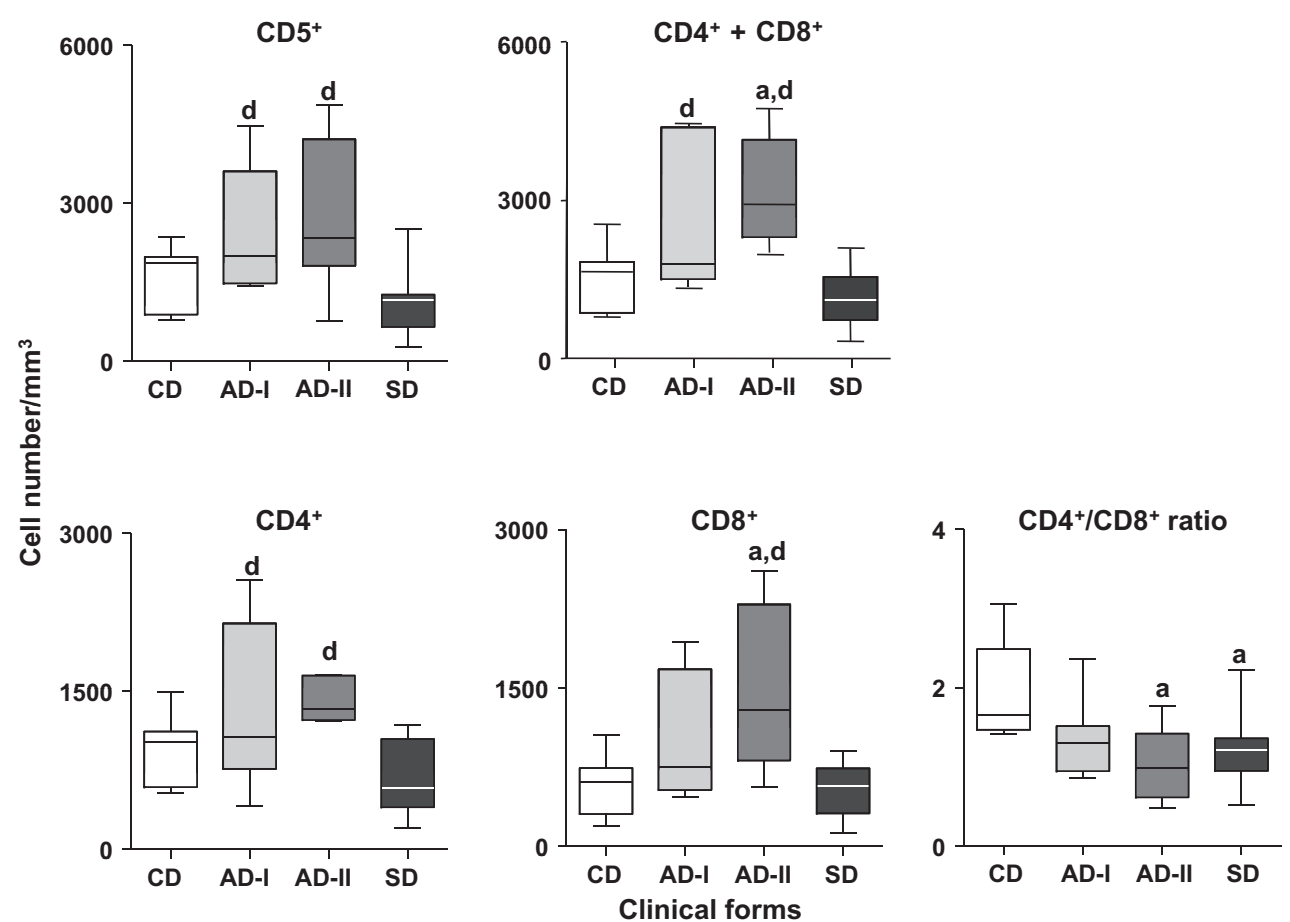

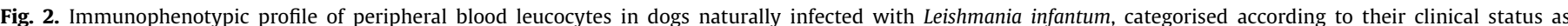

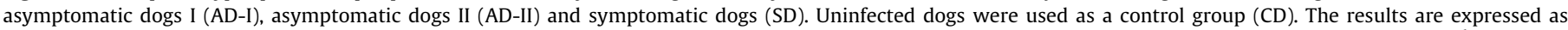

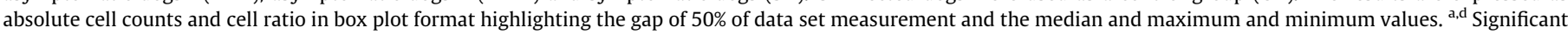
differences at $P<0.05$ compared with $\mathrm{CD}$ and SD, respectively.

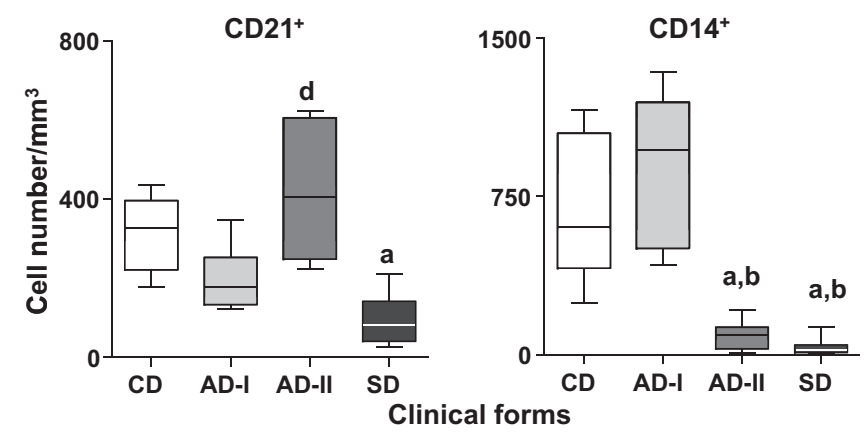

Fig. 3. Immunophenotypic profile of B lymphocytes and monocytes in peripheral blood of dogs naturally infected with Leishmania infantum, categorised according to their clinical status as asymptomatic dogs I (AD-I), asymptomatic dogs II (AD-II) and symptomatic dogs (SD). Uninfected dogs were used as a control group (CD). The results are expressed as absolute cell counts and cell ratio in box plot format highlighting the gap of $50 \%$ of data set measurement and the median and maximum and minimum values. ${ }^{\mathrm{a}, \mathrm{b}, \mathrm{d}}$ Significant differences at $P<0.05$ compared with $\mathrm{CD}, \mathrm{AD}-\mathrm{I}$ and SD, respectively.

AD-I and AD-II had similar total concentrations of $\mathrm{CD}^{+} \mathrm{T}$ lymphocytes, with the main contribution being from $\mathrm{CD}^{+} \mathrm{T}$ lymphocytes, whereas $\mathrm{CD}^{+} \mathrm{T}$ lymphocytes were only high in AD-II. The lower $\mathrm{CD}^{+}: \mathrm{CD}^{+}$ratio in $\mathrm{AD}-\mathrm{II}$ and SD suggests a distinct cellular immune response after seroconversion and disease progression. A more intense parasite load in AD-II and SD may augment the production of $\mathrm{CD}^{+} \mathrm{T}$ lymphocytes and polyclonal immunoglobulin activation, resulting in increased concentrations of IgG. Progressive disease in CVL is associated with suppression of cell-mediated immunity (Pinelli et al., 1994; Reis et al., 2009, 2010). Increased levels of $\mathrm{CD}^{+} \mathrm{T}$ lymphocytes are evident in asymptomatic dogs with a low parasite load (Reis et al., 2006b; Guerra et al., 2009). The absolute number of $\mathrm{CD}^{+} \mathrm{T}$ lymphocytes could be used to discriminate between AD-I and AD-II. However, further studies are require to confirm this hypothesis.

Concentrations of $\mathrm{CD} 21^{+} \mathrm{B}$ lymphocytes were increased in AD-II but decreased in SD, as described previously (Reis et al., 2006b, 2009; Giunchetti et al., 2008). Analysis of lymphoid organs from dogs naturally infected with $L$. infantum revealed hyperplasia of B cells, mainly plasma cells, associated with increased concentrations of anti-Leishmania antibodies (Martinez-Moreno et al., 1993).

AD-I and CD had an increased frequency of CD14 $4^{+}$monocytes compared with $\mathrm{AD}-\mathrm{II}$ and $\mathrm{SD}$, suggesting that higher counts of CD1 $14^{+}$monocytes in AD-I could be important in the control of tissue parasite load and the establishment of resistance mechanisms during ongoing CVL.

\section{Conclusions}

AD-I had elevated counts of circulating eosinophils, CD14 monocytes and $\mathrm{T}$ lymphocytes, particularly $\mathrm{CD} 4^{+} \mathrm{T}$ lymphocytes, whereas $\mathrm{AD}$-II had higher counts of T lymphocytes mainly due to increased $\mathrm{CD}^{+} \mathrm{T}$ lymphocytes and accompanied by a decreased $\mathrm{CD}^{+}: \mathrm{CD}^{+}$ratio. AD-I exhibit a resistance phenotype, whereas AD-II exhibit a susceptibility phenotype.

\section{Conflict of interest statement}

None of the authors of this paper has a financial or personal relationship with other people or organisations that could inappropriately influence the content of the paper.

\section{Acknowledgements}

This work was supported by Fundação de Amparo à Pesquisa do Estado de Minas Gerais, Brazil (FAPEMIG Grant: CBB - APQ-30734.01/07), Programa de Pesquisa para o SUS (PPSUS/MS/CNPq/FAP- 
EMIG/SES-MG/Grant CBB-APQ-00356-10), Conselho Nacional de Pesquisa (CNPq Grant: 472554/2007-7) and Departamento de Ciência e Tecnologia do Ministério da Saúde (DECIT/MS/CNPq/BR/ Grant: 576062/2008-1). ATC, CMC, RCO, OAMF, MC and ABR are thankful to CNPq for the PQ fellowship program.

\section{References}

Andrade, H.M., Reis, A.B., dos Santos, S.L., Volpini, A.C., Marques, M.J., Romanha, A.J., 2006. Use of PCR-RFLP to identify Leishmania species in naturally-infected dogs. Veterinary Parasitology 140, 231-238.

Boceta, C., Alonso, C., Jimenez-Ruiz, A., 2000. Leucine rich repeats are the main epitopes in Leishmania infantum PSA during canine and human visceral leishmaniasis. Parasite Immunology 22, 55-62.

Bourdoiseau, G., Bonnefont, C., Hoareau, E., Boehringer, C., Stolle, T., Chabanne, L., 1997. Specific IgG1 and IgG2 antibody and lymphocyte subset levels in naturally Leishmania infantum-infected treated and untreated dogs. Veterinary Immunology and Immunopathology 59, 21-30.

Cardoso, L., Schallig, H.D., Cordeiro-da-Silva, A., Cabral, M., Alunda, J.M., Rodrigues, M., 2007. Anti-Leishmania humoral and cellular immune responses in naturally infected symptomatic and asymptomatic dogs. Veterinary Immunology and Immunopathology 117, 35-41.

Cavaliero, T. Arnold, P., Mathis, A., Glaus, T., Hofmann-Lehmann, R., Deplazes, P. 1999. Clinical, serologic, and parasitologic follow-up after long-term allopurinol therapy of dogs naturally infected with Leishmania infantum. Journal of Veterinary Internal Medicine 13, 330-334.

da Costa-Val, A.P., Cavalcanti, R.R., de Figueiredo Gontijo, N., Michalick, M.S., Alexander, B., Williams, P., Melo, M.N., 2007. Canine visceral leishmaniasis: Relationships between clinical status, humoral immune response, haematology and Lutzomyia (Lutzomyia) longipalpis infectivity. The Veterinary Journal 174 , 636-643.

Deane, L.M., Deane, M.P., 1962. Visceral leishmaniasis in Brazil. Geographica distribution and transmission. Revista do Instituto de Medicina Tropical de São Paulo 4, 149-212.

Degrave, W., Fernandes, O., Campbell, D., Bozza, M., Lopes, U., 1994. Use of molecular probes and PCR for detection and typing of Leishmania - a minireview. Memorias do Instituto Oswaldo Cruz 89, 463-469.

Desjeux, P., 2001. Worldwide increasing risk factors for leishmaniasis. Medical Microbiology and Immunology 190, 77-79.

Desjeux, P., 2004. Leishmaniasis: Current situation and new perspectives. Comparative Immunology, Microbiology and Infectious Diseases 27, 305-318.

Genaro, O., Raso, P., da Costa, C.A., Carvalho, M.D., do Amaral, F., Botelho, A.C., Williams, P., Dias, M., Mayrink, W., 1992. Montenegro skin tests in dogs experimentally infected with Leishmania (Viannia) braziliensis. Memorias do Instituto Oswaldo Cruz 87, 163-164.

Giunchetti, R.C., Martins-Filho, O.A., Carneiro, C.M., Mayrink, W., Marques, M.J. Tafuri, W.L., Correa-Oliveira, R., Reis, A.B., 2008. Histopathology, parasite density and cell phenotypes of the popliteal lymph node in canine visceral leishmaniasis. Veterinary Immunology and Immunopathology 121, 23-33.

Guerra, L.L., Teixeira-Carvalho, A., Giunchetti, R.C., Martins-Filho, O.A., Reis, A.B., Correa-Oliveira, R., 2009. Evaluation of the influence of tissue parasite density on hematological and phenotypic cellular parameters of circulating leukocytes and splenocytes during ongoing canine visceral leishmaniasis. Parasitology Research 104, 611-622.

Lachaud, L., Chabbert, E., Dubessay, P., Dereure, J., Lamothe, J., Dedet, J.P., Bastien, P., 2002. Value of two PCR methods for the diagnosis of canine visceral leishmaniasis and the detection of asymptomatic carriers. Parasitology 125 197-207.

Lemos, E.M., Laurenti, M.D., Moreira, M.A., Reis, A.B., Giunchetti, R.C., Raychaudhuri, S., Dietze, R., 2008. Canine visceral leishmaniasis: Performance of a rapid diagnostic test (Kalazar Detect) in dogs with and without signs of the disease. Acta Tropica 107, 205-207.

Mancianti, F., Gramiccia, M., Gradoni, L., Pieri, S., 1988. Studies on canine leishmaniasis control. 1. Evolution of infection of different clinical forms of canine leishmaniasis following antimonial treatment. Transactions of the Roya Society of Tropical Medicine and Hygiene 82, 566-567.

Martin-Sanchez, J., Lopez-Lopez, M.C., Acedo-Sanchez, C., Castro-Fajardo, J.J., Pineda J.A., Morillas-Marquez, F., 2001. Diagnosis of infections with Leishmania infantum using PCR-ELISA. Parasitology 122, 607-615.

Martinez-Moreno, A., Martinez-Cruz, M.S., Blanco, A., Hernandez-Rodriguez, S., 1993. Immunological and histological study of T- and B-lymphocyte activity in canine visceral leishmaniosis. Veterinary Parasitology 51, 49-59.
Marzochi, M.C., Coutinho, S.G., De Souza, W.J., De Toledo, L.M., Grimaldi Jr., G., Momen, H., Pacheco Rda, S., Sabroza, P.C., De Souza, M.A., Rangel Jr., F.B., Tramontano, N.C., 1985. Canine visceral leishmaniasis in Rio de Janeiro, Brazil. Clinical, parasitological, therapeutical and epidemiological findings (19771983). Memorias do Instituto Oswaldo Cruz 80, 349-357.

Molina, R., Amela, C., Nieto, J., San-Andres, M., Gonzalez, F., Castillo, J.A., Lucientes, J., Alvar, J., 1994. Infectivity of dogs naturally infected with Leishmania infantum to colonized Phlebotomus perniciosus. Transactions of the Royal Society of Tropical Medicine and Hygiene 88, 491-493.

Oliva, G., Scalone, A., Foglia Manzillo, V., Gramiccia, M., Pagano, A., Di Muccio, T., Gradoni, L., 2006. Incidence and time course of Leishmania infantum infections examined by parasitological, serologic, and nested-PCR techniques in a cohort of naive dogs exposed to three consecutive transmission seasons. Journal of Clinical Microbiology 44, 1318-1322.

Paltrinieri, S., Solano-Gallego, L., Fondati, A., Lubas, G., Gradoni, L., Castagnaro, M., Crotti, A., Maroli, M., Oliva, G., Roura, X., Zatelli, A., Zini, E., 2010. Guidelines for diagnosis and clinical classification of leishmaniasis in dogs. Journal of the American Veterinary Medical Association 236, 1184-1191.

Pinelli, E., Killick-Kendrick, R., Wagenaar, J., Bernadina, W., del Real, G., Ruitenberg, J., 1994. Cellular and humoral immune responses in dogs experimentally and naturally infected with Leishmania infantum. European Journal of Immunology 62, 229-235.

Reis, A.B., Carneiro, C.M., Carvalho, M.G., Teixeira-Carvalho, A., Giunchetti, R.C. Mayrink, W., Genaro, O., Correa-Oliveira, R., Martins-Filho, O.A., 2005. Establishment of a microplate assay for flow cytometric assessment and it is use for the evaluation of age-related phenotypic changes in canine whole blood leukocytes. Veterinary Immunology and Immunopathology 103, 173-185.

Reis, A.B., Martins-Filho, O.A., Teixeira-Carvalho, A., Carvalho, M.G., Mayrink, W., Franca-Silva, J.C., Giunchetti, R.C., Genaro, O., Correa-Oliveira, R., 2006a. Parasite density and impaired biochemical/hematological status are associated with severe clinical aspects of canine visceral leishmaniasis. Research in Veterinary Science 81, 68-75.

Reis, A.B., Teixeira-Carvalho, A., Giunchetti, R.C., Guerra, L.L., Carvalho, M.G., Mayrink, W., Genaro, O., Correa-Oliveira, R., Martins-Filho, O.A., 2006b. Phenotypic features of circulating leucocytes as immunological markers for clinical status and bone marrow parasite density in dogs naturally infected by Leishmania chagasi. Clinical and Experimental Immunology 146, 303-311.

Reis, A.B., Teixeira-Carvalho, A., Vale, A.M., Marques, M.J., Giunchetti, R.C., Mayrink, W., Guerra, L.L., Andrade, R.A., Correa-Oliveira, R., Martins-Filho, O.A., 2006c. Isotype patterns of immunoglobulins: Hallmarks for clinical status and tissue parasite density in Brazilian dogs naturally infected by Leishmania (Leishmania) chagasi. Veterinary Immunology and Immunopathology 112, 102-116.

Reis, A.B., Martins-Filho, O.A., Teixeira-Carvalho, A., Giunchetti, R.C., Carneiro, C.M., Mayrink, W., Tafuri, W.L., Correa-Oliveira, R., 2009. Systemic and compartmentalized immune response in canine visceral leishmaniasis. Veterinary Immunology and Immunopathology 128, 87-95.

Reis, A.B., Giunchetti, R.C., Carrillo, E., Martins-Filho, O.A., Moreno, J., 2010. Immunity to Leishmania and the rational search for vaccines against canine leishmaniasis. Trends in Parasitology 169, 240-249.

Rodriguez-Cortes, A., Fernandez-Bellon, H., Ramis, A., Ferrer, L., Alberola, J., SolanoGallego, L., 2007. Leishmania-specific isotype levels and their relationship with specific cell-mediated immunity parameters in canine leishmaniasis. Veterinary Immunology and Immunopathology 116, 190-198.

Romero, G.A., Boelaert, M., 2010. Control of visceral leishmaniasis in Latin America a systematic review. PLoS Neglected Tropical Diseases 4, e584.

Rosário, E.Y., Genaro, O., Franca-Silva, J.C., da Costa, R.T., Mayrink, W., Reis, A.B., Carneiro, M., 2005. Evaluation of enzyme-linked immunosorbent assay using crude Leishmania and recombinant antigens as a diagnostic marker for canine visceral leishmaniasis. Memórias do Instituto Oswaldo Cruz 100, 197-203.

Solano-Gallego, L., Llull, J., Ramos, G., Riera, C., Arboix, M., Alberola, J., Ferrer, L., 2000. The Ibizian hound presents a predominantly cellular immune response against natural Leishmania infection. Veterinary Parasitology 90, 37-45.

Solano-Gallego, L., Morell, P., Arboix, M., Alberola, J., Ferrer, L., 2001. Prevalence of Leishmania infantum infection in dogs living in an area of canine leishmaniasis endemicity using PCR on several tissues and serology. Journal of Clinical Microbiology 39, 560-563.

Teixeira Neto, R.G., Giunchetti, R.C., Carneiro, C.M., Vitor, R.W., Coura-Vital, W., Quaresma, P.F., Ker, H.G., de Melo, L.A., Gontijo, C.M., Reis, A.B., 2010. Relationship of Leishmania-specific IgG levels and IgG avidity with parasite density and clinical signs in canine leishmaniasis. Veterinary Parasitology 169, 248-257.

Volpini, A.C., Passos, V.M., Oliveira, G.C., Romanha, A.J., 2004. PCR-RFLP to identify Leishmania (Viannia) braziliensis and L. (Leishmania) amazonensis causing American cutaneous leishmaniasis. Acta Tropica 90, 31-37. 